\title{
Interaction research on the online education under the environment of large data base
}

\author{
Xinxin wang ${ }^{1, a}$ \\ ${ }^{1}$ Changchun University of science and technology, Changchun, Jilin Province, China \\ a191720@qq.com
}

Keywords: Big data, Data environment, Online education, Interactive research

\begin{abstract}
The emergence of cloud computing and big data technology, facing a large amount of data generated in the online education interaction, that can realize the identification, analysis, mining and organization structure, at the meantime that can implicit in the learner interaction in the process of unstructured data. The development of the interactive process value of the data was aspirations and predicted learning support service and trend. The guideline was to improve and broaden the online education service, achieve the two-way balance online education service and the needs of learners. Based on the analysis of the interaction bottleneck, demand of online education, and build an interactive platform model with the support of big data and cloud computing, this paper will focus on the interactive platform, online interactive activities to online learners and teachers can achieve the perfection, and the interactive in-depth data mining analysis, solves the problems currently faced by online education.
\end{abstract}

\section{Introduction}

Online education interaction is divided into individual interaction and social interaction, the former is the interaction of learners and learning materials. The latter is the interaction of learners and teachers or learners asks, social interaction is the key factor to improve the level of online education interaction [1]. With the increase of the interactive information resources of online education, online learners and online teachers need to develop and improve, there are many problems in online education.

The interactive platform for online education is lack of unified identity authentication system, the need for repeat identity verification, to cause inconvenience to the user while increasing security risks to the system; lack of unified display applications, the user information is scattered in various applications, and the service function of repeated stack waste; use method, interface and quality is not uniform, and used to the platform maintenance inconvenience [1]. The fragmentation trend of online learning time of terminal equipment on the learner's request is very high, resulting in the decline in the quality of learning; lack of cross terminal resource sharing system, affecting the efficiency and satisfaction of learning, learning to change the terminal, increase the data loss, virus attacks and other security issues.

With the online learners and teachers online service demand of individuation and specialization, the service quality is more and more valued, online education platform must make corresponding strategies according to online learners and online teachers demand change, to adapt to change and improve service demand [1]. Because online education is the Internet based learning, between students and learning resources, teachers and students, students and students communicate through the network is full, so through the analysis of learning mining online learners and teachers demand. The amount of information resources of online education is increasing, the main data resources including structured and unstructured information, and related information within the online education platform based on the data and every day, dominated by a large number of unstructured data and heterogeneous data resources. But at present, the recording, storage and statistical techniques of these heterogeneous data and unstructured data cannot meet the needs of online education interactive platform. Therefore, the structured platform to generate unstructured, heterogeneous data analysis and the potential value of the depth of excavation is inevitable, the 
analysis results fast, timely, efficient and safe information feedback to researchers and teachers for online learning.

\section{Online education interactive platform application of cloud computing and big data}

Cloud computing and big data and interactive platform for online education. Cloud computing is the processing ability through Internet giant, will be processed automatically split into numerous smaller subroutines, by searching, calculating and analyzing the huge pay system is composed of multiple servers, and finally the results will be sent back to the user. Big data technology is an advanced technology of data analysis, from various types of data, the ability to quickly obtain valuable information, is the need for new processing mode to achieve greater decision-making power, insight discovery and process optimization capabilities of massive, high rates of growth and diversification of information assets. Cloud computing emphasizes the ability of dynamic computing which the big data focus on the static calculation of objects [2]. Cloud computing makes up the biggest problem the storage and operation of large data, is to provide a computing platform, and big data using distributed processing methods applied to this platform, the two are complementary to each other.

Cloud computing and the combination of large data applications, the former emphasizes computing power, the latter value storage capacity. Large data processing capability of large amounts of complex data, including data collection, collation, conversion, statistics, the powerful computing capability, and cloud computing requires a large amount of data as the basic operation, so the combination of the two is an inevitable trend [2]. In practical applications, the emergence of cloud computing and the rise of the extensive application of large data, and the combination of big data and cloud computing applications in many areas, has been extended to the field of public problems. But there is no access to the online education industry.

With the advantages of cloud computing, need to consider the online learning online education platform for interactive terminal equipment without the user operation, storage and load capacity, can better realize the sharing of educational resources and education network collaboration, improve the rate and efficiency of education resource utilization greatly. Cloud storage shield data loss, virus and other issues, to protect the privacy of data security and user information, online education platform is the most secure and reliable data storage center. User's access to educational resources and access to educational resources online learning and exchange will generate and accumulate a large number of structured and unstructured data, not only the body mass and growth rate is very fast [2]. Unstructured data has accounted for more than $80 \%$ of the total amount of data, but the current data analysis and processing algorithms and software cannot meet the requirements of unstructured data processing. It can be complex and massive data online education platform for high speed real-time processing of large data technology, change, as the online education platform real-time insight into the learners grasp the needs of learners and provide support to improve the quality of education. The online education platform on a large number of irrelevant information, in-depth analysis of the complex, provide prediction for future education demand trend, this is the online education application of big data and the traditional online education is essentially different.

Application model analysis of online education interaction platform based on cloud computing and big data. According to the above analysis, with the increasing homogenization of online education, online education institutions need to protect rich educational resources and high quality at the same time, online learners and teacher's preference analysis better, for each platform for users to provide the personalized service [3].

Application of cloud computing and big data technology online education interactive platform is mainly divided into user application service layer, data resource processing layer, infrastructure hardware layer three parts, in order to analyze the function of the three parts.

User service application layer. Service application layer is the window of the interaction of resources, is the bridge of the user's use of resources, and the convenience of the user experience has a great relationship [3]. Therefore, the application service layer is based on user requirements, the 
information resources request reconstruction and provide, to achieve the classification of information resources, users enjoy personalized service resources.

Platform for each user to show the content is not the same. For teachers, the online learning platform for real-time feedback and analysis results, especially the analysis of the learning style and preference, real-time tracking of students before and after class, class and the completion of the course, the students feedback on the platform behavior, learning records intelligent tracking analysis. For learners, constitute learning, question answering, evaluation, interactive learning mode four, the use of rich learning resources, according to the background of the data mining. Provide learning schedule and personalized learning program [3].

Online education interactive platform users see is good service interface, no need to know the background of data integration process, fully completed by the data processing layer of the platform, so have a better user experience [3]. Platform is the distribution center of all teaching resources, integration of resources to facilitate unified management and use of the same resource, just save one, through the resources associated, can be quickly called in any system. At the same time to avoid the sharp increase in database, greatly reduce the network load, reduce the working time of users and platforms, and maintain the uniqueness of resources, the use of the resources of the application of the resource changes are automatically updated.

Data resource processing layer. In the face of complex data increase rapidly, online education interaction platform based on cloud computing and big data for modern data management, support all data types, such as files, pictures, video, blog, click on the flow and the geographical spatial data, and to "cloud storage" permanently stored in the data center, updated data, implementation data sharing, analysis, discovery, data integration and optimization, enhance the value of data [4].

Using load balancing advantages, effective and transparent to expand the bandwidth of network equipment and servers, increase the amount of leaf swallow online education platform, interactive platform to strengthen network data processing ability, improve service flexibility and availability [4]. In the face of the user or a large number of concurrent access to data flow, can be shared to multiple devices, reduce the teacher and the learner waits for the response time; at the same time to do parallel processing, processing results back to the online interactive platform, platform system processing capacity has been greatly improved.

Offline data is the information resources in the database, which is collected by the server, the client and the proxy server. The data are processed with big data technology to remove unwanted data, using cluster analysis and classification algorithm for processing data for pattern analysis, the establishment of data resources for data mining analysis [5]. Online data is due to data flow dynamics and flow characteristics, in the realization of data stream mining, the inflow of data flow, do less memory using cloud computing, fast processing, mining association rules, classification and clustering.

The integration of data is off-line data as the sample library reference for online data analysis, timely feedback results, and with the passage of time and the user's demand for information resources change, the analysis results to update the resource. Through the data mining process, data filtering, analysis and integration, the establishment of multi resource classification results, in accordance with the different needs of users to make decisions, to form an index for user access and use of services to facilitate.

Integration of data is mainly for the integration of the user to do preparation, the user's information resources for similarity analysis, similar to the user classification, the allocation of similar information resources [5]. According to the basic information, online learning style, learning satisfaction and perception of the four dimensions of learning services, users can achieve the customized service, personalized service, accurate service, convenient for the user to facilitate the extraction of the resources they need to. In the end, the user needs the resources according to different authorization. Do unified standardized treatment. Upload to the service application layer, display in the user interface. 


\section{The advantage based on cloud computing and big data online education interactive platform application}

Cloud computing and big data combined with the development of online education has tremendous simulative effect, is not only to provide services, but also promote the development of education, enhance the core competitiveness of online education, maintain the healthy development of online education.

To achieve personalized precision services for different users. In the process of building a personalized online education platform based on the core of the resources, the most important change is to provide different personalized services for different users. And the application of cloud computing and big data is to strengthen the analysis and utilization of online education platform user research and interactive data. Improve service content; improve the quality of personalized service, complete the platform for the user's tracking service, accurate service, knowledge related services and promotion services [6]. With the rapid growth of data platform, extract valuable information, real-time analysis of user feedback, model of different categories for different users, to provide targeted services, enhance the user experience, and improve the quality of service. Even if the analysis of the same data source, but because of different objects, the analysis results will be different, the service provided is also different, so accurate service. Even similar users, for different individuals, analysis of different data sources, the results are different, the services provided are not the same, so personalized service.

The trend of educational development and the change of hot spots. Through the big data and cloud computing technology, changes the situation of the passive update teaching resources, according to the needs of online learners to actively update resources, enhance the role of online education platform [7]. Not only to provide online learners with the need of learning resources, but also for online teachers to provide more quality of teaching resources and research basis. Interactive online education platform through collecting and sorting, analysis, mining depth and summary of user data, analysis of the changes of development in the field of education and focus on the macroscopic, faster insight into the latest progress toward the learners 'interest, and related content, more new platform for online learning resources, and ensure the real-time leading and learning content.

Providing unlimited data storage capacity and more reliable data security. With the development of the interactive development of online education, the amount of information data is growing rapidly, resulting in a large number of semi-structured and unstructured information. The emergence of cloud computing data storage and computing has been solved, the way of distributed storage can continue to collect a large number of data, will not cause the problem of storage space. Interactive platform for online education, cloud storage, to ensure the reliability of storage data, and can be updated in real time, to effectively solve the massive data resources, such as query, management and other issues [7]. The use of cloud computing data fault-tolerant, multi copy equipment such as interchangeable isomorphism means to protect the security of data storage platform. Data is stored in the cloud, will not be caused by computer viruses or hard disk damage caused by data loss. At the same time the liberation of the user requirements for terminal equipment.

Improving the management ability of the interactive platform for online education. Application of cloud computing and big data online education interactive platform, to be able to face the specific application of data needs, to achieve fast, timely and effective response [8]. According to the change and growth of demand, the platform has a good performance to expand the space and expansion of the stability and reliability of the support, efficient processing of a variety of types of data. On this platform, the online education institutions use the advantages of cloud computing technology and big data, and fully tap the value of their own data, and realize the transformation from cost center to profit center. Through the integration of data assets, standardization of data assets, the formation of flexible and extensible and easy to update, control, isolation, environmental protection and efficient analysis of data management platform, implementation support standards development, user service, diversified development of a variety of application support mode, the formation of loose coupling, basic data and application data heterogeneous two level data management level [8]. At the same time, online educational institutions can manage their data assets, to enhance the data 
management capabilities of the platform, try to get to the online learners and online teachers insight into the development of online education to drive data.

\section{Summary}

For the important research field of online education interaction, the emergence of cloud computing and big data technology not only affects the form of online education interaction, but also provides a new way of thinking and means for interactive information analysis. On the one hand, new technology makes the interactive behavior of continuous towards real-time and fragmentation of the direction of development, make the interactive process more complex; on the other hand, new technology is making access to a large number of interactive data, especially the possible behavioral data, and thus have more understanding of online learning process and teaching decision-making. Online education interaction as information service, although online education interaction platform has the advantages of resources, but the disadvantage in technology and other aspects also limits its own development. This paper constructs a model of online education platform based on big data and cloud computing support, functional analysis platform, online interactive activities to online learners and teachers can achieve the perfect, summed up the advantages of using online education interactive platform for cloud computing and big data. Therefore, the rapid development of online education interactive platform requires the use of cloud computing and big data innovation to enhance the core competitiveness of online education interaction.

\section{References}

[1] J.F. Luo nd Xu Fei. The university information framework of big data era, China education information, 2014, vol.3, pp.11-13

[2] Q.Y. Li and J.P. Zhu, The impact of big data on College Teaching, China University teaching, 2014, vol.9, pp.41-44.

[3] X.L. Jin and X.Q. Cheng, Journal of systems and big data analysis techniques, Software education 2014, vol.9, pp.17-19.

[4] Y.P. Zhang and Y.T. Li, The study and analysis of MOOCs based big data and educational survey , Tsinghua University education research, 2013, vol.4, pp.22-26.

[5] Y.T. Zhang and X.F. Liao, Research and development of big data base of education, Computer application data, 2013, vol.5, pp. 16-22.

[6] X.F. Feng and M.H.Yu, Study and analysis system architecture based on big data technology, Journal of East China Normal University, 2014, vol.2, pp.20-29.

[7] Q.J. Chen, Study on the change of teaching mode under the background of big data, China education information technology, 2013, vol.4, pp.15-18.

[8] D.Y. Ma, A review of the large data visibility analysis, Journal of software, 2014, vol.5, pp.19-23. 